\title{
Correction to: Identification and evaluation of natural organosulfur compounds as potential dual inhibitors of a-amylase and a- glucosidase activity: an in-silico and in-vitro approach
}

\author{
Parvej Ahmad $^{1}$ - Sahir Sultan Alvi ${ }^{1}$ Johar Iqbal ${ }^{2}$. M. Salman Khan $\mathbb{1}^{1}$
}

Published online: 22 October 2021

(c) Springer Science+Business Media, LLC, part of Springer Nature 2021

Correction to: Medicinal Chemistry Research (2021) https://doi.org/10.1007/s00044-021-02799-2

In this article, the affiliation details for corresponding author M. Salman Khan were incorrectly given as 'Department of Biochemistry, Faculty of Medicine, Jazan
University, Jazan, Kingdom of Saudi Arabi' but should have been ' IIRC-5, Clinical Biochemistry and Natural Product Research Lab, Department of Biosciences, Integral University, Lucknow, UP 226026, India.

The original article has been corrected.

These authors contributed equally: Parvej Ahmad, Sahir Sultan Alvi

M. Salman Khan

contactskhan@gmail.com

1 IIRC-5, Clinical Biochemistry and Natural Product Research Lab, Department of Biosciences, Integral University, Lucknow, UP 226026, India

2 Department of Biochemistry, Faculty of Medicine, Jazan University, Jazan, Kingdom of Saudi Arabia 